\title{
Der Temperaturkoeffizient der elektrischen Doppelbrechung in Flüssigkeiten.
}

Von Nikolaus Lyon in Freiburg i. B.

(Eingegangen am 15. Oktober 1921.)

C. Bergholm I) hat kürzlich unter dem obigen Titel eine Untersuchung veröffentlicht, deren Resultate eine willkommene Ergänzung unserer diesbezüglichen Messungen ${ }^{2}$ ) bilden. An beiden Arbeiten hat nun kürzlich Peter Lertes ${ }^{3}$ ) eine Kritik geübt, anf die ich mit einigen Worten näher eingehen möchte.

Zur Erklärung der elektrischen Doppelbrechung stehen zurzeit drui Theorien zur Diskussion:

1. Die sogenannte Orientieruugstheorie von Langevin ${ }^{4}$ ), nach der sich die Moleküle in einem elektrischen Felde polarisieren und richten. Nach Langevin läßt sich die Kerrkonstante $B$ der elektrischen Doppelbrechung auf dic Form bringen:

$$
B=C \cdot \frac{(\varepsilon+2)^{2} \cdot\left(n^{2}+2\right)^{2} \cdot d}{T \cdot n}
$$

wo $\varepsilon$ die Dielektrizitätskonstante, $n$ der Brechungsexponent, $d$ die Dichte, $T$ die absolute Temperatur ist und wo in $C$ alle von $T$ unabhängigen Größen zusammengefaßt sind.

2. Born') hat diese Thoorie erweitert unter Berücksichtigung der Reinganum-Debyeschen Dipole. Diese Dipole sollen durch die äußeren Felder und durch die Temperatur nicht merklich beeinflußt werden. Nach Born kann man schreiben:

$$
B=\frac{\text { const } .(\varepsilon+2)^{2} \cdot\left(n^{2}+2\right)^{2}}{n}\left(\frac{2}{K T}+\frac{M^{2}}{K^{2} T^{2}}\right)
$$

oder wenn wir die Gleichung passend umformen:

$$
B_{(\text {(Born })}=B_{(\text {Langevin })} \cdot\left(1+\frac{M^{2}}{K \cdot T \cdot \mathscr{B}}\right)
$$

Bei Anwesenleit von Dipolen mu also die Kerrkonstante mit fallender Temperatur stärker ansteigen, als nach Langevin zu erwarten ist. Das zweite Klammerglied von (3) stellt den Anteil der Dipole an der elektrischen Doppelbrechung dar.

1) C. Bergholm, Ann. d. Phys. 65, 128, 1921.

2) N. Lyon und F. Wolfram, Ann. d. Phys. 63, 739, 1920.

3) P. Lertes, ZS. f. Phys. 6, 257, 1921.

1) P. Langevin, Le Radium 7, 249, 1910.

5) M. Born, Ann. d. Phys. 55, 17i, 1918. 
Nikolaus Lyon, Der Temperaturkoeffizient der elektr. Doppelbrechung usw. 65

Sind keine Dipole vorhanden, so ist dieses Glied Null und die Bornsche Formel geht in die von Langevin über. Sind Dipole vorhanden, so maß dieses Glied stets positir sein, ganz gleichgültig, ob es sich um einen Körper mit positiver oder negativer elektrischer Doppelbrechung handelt 1 ). Bei Vorhandensein von Dipolen muB also der Zahlenwert von $B$ (Born) stets größer sein als der von Langevin ").

3. Gans${ }^{2}$ ) und auf anderem Wege Lundblad ${ }^{3}$ ) haben den Langevinschen Ansatz weiter entwickelt nnter der Annahme, daB die fertigen Momente durch äußere Felder und Temperatur merklich beeinflußt werden, sie fordern eine noch stärkere Temperaturabhängi@̣keit der Kerrkonstante als Born.

Herr Lertes hat nun darauf hingewiesen, daB die seinerzeit von Wolfram und mir veröffentlichten Messungen zur Prüfung der Theorie von Langevin und Born und die daraus gezogenen Schlüsse insofern nicht absolut bindend sind, als einige der in die Formel eingehenden Größen, wie die Dielektrizitätskonstante und vor allem die Dichten. von uns für die tiefen Temperaturen teilwoise extrapoliert werden mußten. Die kürzlich ersehienene Mitteilung von Gans und Isnardi 4) über die Temperaturabhängigkeit der Dielektrizitätskonstante und Messungen von Seitz, Alterthum and Lechner ${ }^{5}$ ) über die Dichten bei tiefen Temperaturen gestatten eine Neuberechnung unserer Resultate. Die erhaltenen Werte weichen von unseren alten nur wenig ab.

Die in den folgenden Tabellen niedcrgelegtei Werte sind nuninehr sämtlich gemessen, ein extrapolierter Wert ist eingeklammert.

Tabelle 1. Schwefelkohlenstolf.

\begin{tabular}{|c|c|c|c|c|c|}
\hline $\begin{array}{c}\text { Temperatur } \\
t^{0}\end{array}$ & $\ddot{*}$ & $n$ & 1 & $\begin{array}{c}\frac{b_{l 0}^{3}}{b_{180}} \\
\text { lier. nach } \\
\text { Langerin }\end{array}$ & $\frac{B_{t^{0}}}{B_{18^{0}}}$ \\
\hline $\begin{array}{r}18^{0} \\
0 \\
-18,5 \\
-37,7 \\
-47,3 \\
-68,3 \\
-78,5\end{array}$ & $\begin{array}{l}2,64 \\
2,70 \\
2,75 \\
2,78 \\
2,79 \\
2,80 \\
2,81\end{array}$ & $\begin{array}{l}1,627 \\
1,641 \\
1,656 \\
1,671 \\
1,679 \\
1,696 \\
1,704\end{array}$ & $\begin{array}{l}1,265 \\
1,291 \\
1,318 \\
1,345 \\
1,359 \\
1,391 \\
1,404\end{array}$ & $\begin{array}{l}1 \\
1,129 \\
1,274 \\
1,444 \\
1,537 \\
1,768 \\
1,897\end{array}$ & $\begin{array}{l}1 \\
1,089 \\
1,205 \\
1,397 \\
1,497 \\
1,708 \\
1,819\end{array}$ \\
\hline
\end{tabular}

1) Herr Iuertes kommit bei Besprechung der Bergholmschen Messungen auf S. 266 (letzter Absatz) infolge irttümlicher Auffassung der Erseheinung zun eutgegengesetzten Schluß.

2) R. Gaus, Ann. d. Pliys. 64, 481, 1921.

3) R. Luudbiad, Diss. Upsala 1920.

4) R. Gans und H. Isnardi, Phys. ZS. 22, 230, 1921 .

5) Seitz, Alterthum u. Iechner, Ann. d. Phys. 49, 91, 1916. Zeitschrift für Physik. Bd. VIII. 
Tabelle 2. Äthylätluer.

\begin{tabular}{|c|c|c|c|c|c|}
\hline $\begin{array}{c}\text { Temperatur } \\
t^{0}\end{array}$ & $\left.\varepsilon^{1}\right)$ & $n$ & $d$ & 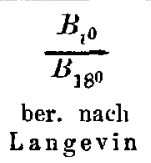 & $\frac{B_{t^{0}}}{B_{18^{0}}}$ \\
\hline $\begin{array}{r}18^{0} \\
0 \\
-21,7 \\
-38,9 \\
-60,5 \\
-78,5\end{array}$ & $\begin{array}{l}4,37 \\
4,68 \\
5,15 \\
5,61 \\
6,31 \\
7,00\end{array}$ & $\begin{array}{l}1,355 \\
1,365 \\
1,378 \\
1,387 \\
1,399 \\
(1,408)\end{array}$ & $\begin{array}{l}0,715 \\
0,736 \\
0,759 \\
0,778 \\
0,800 \\
0,819\end{array}$ & $\begin{array}{c}1 \\
1,215 \\
1,573 \\
1,973 \\
2,689 \\
(3,548)\end{array}$ & $\begin{array}{l}1 \\
1,220 \\
1,547 \\
1,927 \\
2,707 \\
3,880\end{array}$ \\
\hline
\end{tabular}

Tabelle 3. Äthyläther.

\begin{tabular}{c||c|c|c||c|c}
\hline $\begin{array}{c}\text { Trmperatur } \\
t^{0}\end{array}$ & $\left.\varepsilon^{2}\right)$ & $\begin{array}{c}\frac{B_{i 0}}{B_{150}} \\
\text { ber. nach } \\
\text { Langerin }\end{array}$ & $\begin{array}{c}\text { Temperatur } \\
t^{0}\end{array}$ & $\left.\varepsilon^{2}\right)$ & $\begin{array}{c}\frac{B_{i 0}}{B_{180}} \\
\text { her. nach } \\
\text { Langevin }\end{array}$ \\
\hline $18^{0}$ & 4,36 & 1 & $-38,9$ & 5,74 & 2,048 \\
0 & 4,60 & 1,190 & $-60,5$ & 6,50 & 2,821 \\
$-21,7$ & 5,25 & 1,623 & $-78,5$ & 6,97 & $(3,536)$
\end{tabular}

Die beobachteten und nach Langevins Formel berechneten Werte stimmen sowohl für Schwefelkohlenstoff als auch für Äther recht gut iiberein, wobei die berechneten Werte fast durchweg etwas größer als die beobachteten sind, d. h. die Kerrkonstante nimmt mit fallender

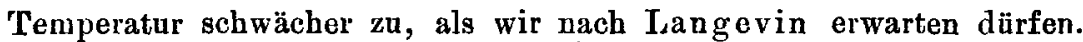
Die Bornsche Korrektur würde die Ubereinstimmung versehlechteru, da die Bornsche Theorie eine stärkere Abnahme der Kerrkonstante als nach Langevin fordert.

Der Berechnung des Langevinwertes $\frac{B_{t^{0}}}{B_{18^{0}}}$ der Tabelle 2 wrorden die Abeggschen Werte der Dielektrizitätskonstante zugrunde gelegt. Tabelle 3 ist auf Grund der Werte von Gans und Isnardi berechnet.

Die eingeklammerten wegen Extrapolation unsicheren Werte bei Athyläther, der nach Debye starke Dipole aufweist, würden die Bornsche Korrektion rechtfertigen. Ihnen kommt aber wegen der Unsicherheit des Wertes von $n$, der in der dritten Potenz eingeht, keine Beweiskraft zn.

Die neuen Messungen von Bergholm bestätigen bis anf Äther unsere Messungen und ergänzen sie nach höheren Temperaturen zu.

1) Dielektrizitätskonstante $\varepsilon$ nach R. Abegg, Wied. Anu. 60, 54, 1897.

2) Dielektrizitätskonstante $\varepsilon$ nach R. Gans und H. Isnardi, l. c. 
Der Temperaturkoeffizient der elektrischen Doppelbrechung in Flüssigkeiten. 67

Bei Schwefelkohlenstoff und Toluol ${ }^{1}$ ) ist die (Ubereinstimmung eine gute, bei Äthyläther weichen allerdings die Resultate zùgunsten der Bornschen Theorie von unseren ab.

Anch hier liegt, glaube ich, ein Miljverständnis in den Darlegungen von Lertes vor.

Das $R^{2}$ ) bei Bergholm entspricht:

$$
R=\left(\frac{1}{B_{20}}\right) \cdot \frac{B_{t^{\prime \prime} \text { beobachtet }}}{B_{t^{\prime \prime} \text { berechnet }}}
$$

nach Bergholm ist nämlich:

$$
R=\frac{B_{t^{\prime \prime}}}{B_{200}} \cdot \frac{n T}{(\varepsilon+2)^{2}\left(n^{2}+2\right) d}
$$

der erste Teil von (5) ist das gemessene $\frac{B_{t^{6}}}{B_{2 a^{\prime}}}$ seiner Tabellen, der zweite Faktor ist aber nach Langevin:

$$
\frac{n T}{(\varepsilon+2)^{2}\left(n^{2}+2\right)^{*} . \delta}=\frac{c}{B_{t^{\prime \prime} \text { berechnet }}}
$$

$R$ ist deshalb nicht dem $Q$ von Born ${ }^{3}$ ) proportional, sondern etwa dem reziproken Wert von $Q$, oder mit anderen Worten: Fält $R$ mit fallender Temperatur, so ist der nach Langevin berechnete Wert bereits größer als der gemessene und würde durch Anbringen der Bornschen Korrektur erst recht größer werden. Nach Born sollte li bei Anwesenheit ron festen Momenten mit fallender Temperatur ansteigen. P. Lertes ${ }^{4}$ ) kommt zum entgegengesetzten Ergebuis, das ich hier wörtlich wiedergebe: "Nimmt also $h$ mit steigender Temperatur zu, so müsseu auf jeden Fall feste Momente vorhanden sein". Die Folgerungen, die Lertes aus den Bergholmschen Messungen zieht, sind jedenfalls nnzutreffend. Bei Bergholm steigt fast durchweg I mit steigender Temperatur.

Freiburg i. B., Physikalisehes Institut ler Universität.

1) N. Lyon u. F. Wolfram, l. c. S. 750 .

2, C. Bergbolm, l. c. S. 182.

3) M. Born, l. c. S. 220.

4) P. Lertes, 1. c. S. 266. 